\title{
MicroRNA Regulation of Endothelial Junction Proteins and Clinical Consequence
}

\author{
Yugang Zhuang, ${ }^{1,2}$ Hu Peng, ${ }^{1}$ Victoria Mastej, ${ }^{2}$ and Weiguo Chen ${ }^{2}$ \\ ${ }^{1}$ Department of Emergency Medicine, Shanghai Tenth People's Hospital, Tongji University, Shanghai, China \\ ${ }^{2}$ Division of Pulmonary, Critical Care, Sleep and Allergy, Department of Medicine, University of Illinois College of Medicine, \\ Chicago, IL, USA \\ Correspondence should be addressed to Weiguo Chen; weiguo@uic.edu
}

Received 10 September 2016; Accepted 3 November 2016

Academic Editor: Yutong Zhao

Copyright (c) 2016 Yugang Zhuang et al. This is an open access article distributed under the Creative Commons Attribution License, which permits unrestricted use, distribution, and reproduction in any medium, provided the original work is properly cited.

Cellular junctions play a critical role in structural connection and signal communication between cells in various tissues. Although there are structural and functional varieties, cellular junctions include tight junctions, adherens junctions, focal adhesion junctions, and tissue specific junctions such as PECAM-1 junctions in endothelial cells (EC), desmosomes in epithelial cells, and hemidesmosomes in EC. Cellular junction dysfunction and deterioration are indicative of clinical diseases. MicroRNAs (miRNA) are 20 nucleotide, noncoding RNAs that play an important role in posttranscriptional regulation for almost all genes. Unsurprisingly, miRNAs regulate junction protein gene expression and control junction structure integrity. In contrast, abnormal miRNA regulation of junction protein gene expression results in abnormal junction structure, causing related diseases. The major components of tight junctions include zonula occluden-1 (ZO-1), claudin-1, claudin-5, and occludin. The miRNA regulation of ZO-1 has been intensively investigated. ZO-1 and other tight junction proteins such as claudin-5 and occludin were positively regulated by miR-126, miR-107, and miR21 in different models. In contrast, ZO-1, claudin-5, and occludin were negatively regulated by miR181a, miR-98, and miR150. Abnormal tight junction miRNA regulation accompanies cerebral middle artery ischemia, brain trauma, glioma metastasis, and so forth. The major components of adherens junctions include VE-cadherin, $\beta$-catenin, plakoglobin, P120, and vinculin. VE-cadherin and $\beta$-catenin were regulated by miR-9, miR-99b, miR-181a, and so forth. These regulations directly affect VE-cadherin- $\beta$-catenin complex stability and further affect embryo and tumor angiogenesis, vascular development, and so forth. miR-155 and miR-126 have been shown to regulate PECAM-1 and affect neutrophil rolling and EC junction integrity. In focal adhesion junctions, the major components are integrin $\beta 4$, paxillin, and focal adhesion kinase (FAK). Integrin $\beta 4$ has been regulated by miR-184, miR-205, and miR-9. Paxillin has been regulated by miR-137, miR-145, and miR-218 in different models. FAK has been regulated by miR-7, miR-138, and miR-135. Deregulation of miRNAs is caused by viral infections, tumorigenesis, and so forth. By regulation of posttranscription, miRNAs manipulate junction protein expression in all cellular processes and further determine cellular fate and development. Elucidation of these regulatory mechanisms will become a new alternative therapy for many diseases, such as cancers and inflammatory diseases.

\section{Introduction}

Cellular junctions are connective structures between cells existing in various tissues, such as epithelium, endothelium, and intestine. Although the cellular junction components and types may vary in different tissues, the majority of components in junctions are similar. Cellular junctions usually have tight junctions, adherens junctions, and focal adhesion junctions. In the endothelium, two identical PECAM-1s also form a junction and stabilize the major junctions. In addition to barrier function, some junction proteins, such as connexins, form a junction between cells which functions as an ion or water exchange channel (connexon). Integrins associate with focal adhesion kinase (FAK) and paxillin to form a focal adhesion complex, anchoring cells on the basal membrane. The focal adhesion complex also functions as a mechanical sensor which transduces extracellular signal into an intracellular downstream signaling pathway. Thus, these cellular junctions regulate cell function in their own ways. 
MicroRNAs (miRNAs) are noncoding RNAs typically $\sim 20$ nucleotides in length and transcribed by RNA polymerase II from individual miRNA genes or coding gene introns. Most miRNAs are associated with RNA-induced silencing complex (RISC) and destabilize or inhibit target mRNA translation via binding to the $3^{\prime}$ untranslated terminal ( $3^{\prime}$ UTR). miRNAs provide precise posttranscriptional control of target gene mRNA. Dysfunctional miRNA causes an abnormally high transcription level of the target mRNA and related clinical consequences. The large number and variable function of miRNA identified to date indicate that miRNAs are involved in a vast array of cellular processes, including development, growth, and tumorigenesis $[1,2]$. However, the importance of miRNAs for cellular junction gene transcription has not been fully characterized.

Here, we review the important role of miRNAs in the processes of endothelial junction protein transcription and clinical relationships. It will help us to better understand the mechanism behind miRNA regulation of endothelial junctions and provide alternative therapies for the diseases caused by endothelial junction abnormality.

\section{Tight Junction Proteins}

2.1. Zonula Occludens-1 (ZO-1)/Occludin/Claudin-5. miRNA regulation of tight junctions has been systemically reviewed [3], and more miRNAs have been found involving tight junction expression. Moreover, the clinical consequence of miRNA regulation has not been reviewed, particularly endothelial tight junction regulation.

miRNA-107 abrogated the amyloid-beta (Abeta) proteininduced disruption of blood-brain barrier (BBB) and endothelial cell dysfunction. 3-UTR assay showed that endophilin$1 \mathrm{mRNA}$ is the direct target of miR-107, which further affected endothelial junction by upregulation of ZO-1, occludin, and claudin-5 [4]. The rat permanent middle cerebral artery occlusion (MCAO) model showed that MCAO significantly increased $\mathrm{BBB}$ permeability in MCAO area. The expression of claudin-5 was decreased in MCA ischemic areas after upregulation of miR-150. Dual-luciferase assay confirmed that miR-150 directly regulated angiopoietin receptor Tie2 and then downregulated claudin-5 [5]. Deletion of miR-150 or knockout miR-150 caused irreversible increase in vascular permeability in mouse and cell model [6]. Interestingly, miR-21 increased in the brain following traumatic brain injury, promoting junction protein expression and exerting a protective effect on BBB by activating Ang-1/Tie2 axis [7].

In vitro and in vivo models showed that miR-98 negatively regulated tight junction proteins, such as ZO-1, by targeting hypoxia-inducible factor-1 (HIF-1) [8]. Diabetes (DM) rats had significant decrease in miR-126, claudin5, occluding, and ZO-1 expression and worsened blood retinal barrier. Niaspan treatment reversed these deleterious effects and enhanced miR-126 level. This observation indicated that miR-126 plays an important role in the Niaspanmediated antidiabetic effect and enhances endothelial barrier by targeting tight junction protein expression [9]. In glioma ECs, upregulation of miR-181a targeted Kruppel-like factor 6 (KLF6), downregulating ZO-1, occluding, and claudin-5, and increased permeability of the blood-tumor barrier (BTB) [10].

Similarly, by targeting runt-related transcriptional factor 1 (RUNX1), miR-18a downregulated mRNA of ZO-1, occludin, and claudin-5 in glioma vascular endothelial cells (GECs) and increased the permeability of BTB [11]. In metastatic breast cancer cells, miR-105 downregulated ZO-1 and increased endothelial barrier permeability, although the underlying mechanism was unclear [12]. By targeting HIV-1 Tat protein C (HIV-1 Tat C), miR-101 downregulated VE-cadherin, further downregulated claudin-5, and changed BMVECs permeability [13] (Table 1).

\section{Adherens Junction Proteins}

3.1. VE-Cadherin. The major components of adherens junctions include cadherins, $\beta$-catenin, plakoglobin, p120, and vinculin. These adherens proteins play an important role not only in cellular connection, but also in cellular signal exchange, contact-induced growth inhibition, and so forth [14]. Adherens junctions exist in all types of cellular connections and are relatively stable. As a major component of adherens junctions, E-cadherin (CDH1, existing in epithelium), VE-cadherin (CDH5, existing in vascular endothelium), and $\mathrm{N}$-cadherin ( $\mathrm{CDH} 2$, existing in neurons) have been extensively studied in past decades and miRNA regulation has been reported. miR-9 downregulated E-cadherin accompanying tumor metastasis or osteoblast differentiation $[15,16]$.

Early studies showed that miR-99b, miR-181a, and miR$181 \mathrm{~b}$ potentiated the mRNA and protein expression of PECAM- 1 and VE-cadherin accompanying differentiation of human embryonic stem cells to vascular endothelial cells [17]. miR-21 also regulated induced pluripotent stem cell (iPSC) differentiation into ECs by directly targeting VE-cadherin [18]. miR-125b inhibited VE-cadherin translation and in vitro tube formation by tumor ECs [19]. Overexpression of miR-142a-3p resulted in loss of vascular integrity, hemorrhage, and vascular remodeling during zebrafish embryonic development, while loss of function of miR-142a-3p caused abnormal vascular remodeling. MiR-142a-3p functions in part by directly repressing VE-cadherin [20]. VE-cadherin also played a role in HIV-associated neurological disorders. HIV-1 Tat protein C increased the expression of miR-101, which led to downregulation of VE-cadherin and further caused an adverse effect on blood-brain barrier integrity and permeability [13]. By directly targeting VE-cadherin, ectopic expression of miR-27a blocked capillary tube formation and angiogenesis [21]. MiR-302c and miR-26b-5p also showed similar function during hepatocarcinoma vascular tube formation $[22,23]$. By regulation of signal pathways, such as TGF- $\beta$ and TGF- $\beta 2$ signal pathways, several miRNAs, such as miR-20a and miR-21, indirectly modulated adherens junction protein expression and endothelial-mesenchymal transition $[18,24]$ (Table 1).

3.2. $\beta$-Catenin. $\beta$-Catenin is not only a central molecule of many signaling pathways, such as the Wnt signaling pathway, but also a critical part of the VE-cadherin junction complex. 
TABLE 1: The major components of EC junction and their targeting miRNAs.

\begin{tabular}{|c|c|c|}
\hline Junction protein & Targeting miRNAs & Clinical consequence \\
\hline ZO-1/occludin/claudin-5 & $\begin{array}{l}\text { miR-105, miR-181a, } \\
\text { miR-18a, miR-21, miR-150, } \\
\text { miR-101, miR-126 }\end{array}$ & $\begin{array}{c}\text { EC barrier disruption } \\
\text { Tumorigenesis }\end{array}$ \\
\hline VE-cadherin & $\begin{array}{c}\text { miR-99b, miR-181a, } \\
\text { miR-181b, miR-21, } \\
\text { miR-125b, miR-142a-3p, } \\
\text { miR-101, miR-27a, } \\
\text { miR-302c, miR-26b-5p, } \\
\text { miR-20a }\end{array}$ & $\begin{array}{l}\text { EC barrier disruption } \\
\text { Tumorigenesis }\end{array}$ \\
\hline$\beta$-Catenin & miR-1826, miR-23b & Growth, apoptosis, invasion \\
\hline PECAM-1 & miR-126, miR-155 & $\begin{array}{c}\text { EC barrier disruption } \\
\text { Tumorigenesis }\end{array}$ \\
\hline Integrin $\beta 4$ & miR-184, miR-205, miR-9 & Tumor metastasis \\
\hline Paxillin & $\begin{array}{l}\text { miR-137, miR-145, miR-218, } \\
\text { miR-125b }\end{array}$ & $\begin{array}{l}\text { Tumor metastasis } \\
\text { Infection }\end{array}$ \\
\hline FAK & miR-135, miR-138, miR-543 & $\begin{array}{l}\text { Tumorigenesis } \\
\text { Tumor invasion }\end{array}$ \\
\hline
\end{tabular}

By association with VE-cadherin, the $\beta$-catenin/VE-cadherin complex plays an important role in maintaining EC junction integrity. By far, of the majority of these studies focused on the role of miRNAs in tumorigenesis or EMT [15, 25]. For example, miR-1826 level was much lower in bladder cancer (BC) cell lines compared to normal bladder cell lines. Transfection of miR-1826 into BC cells inhibited BC cell viability, invasion, and migration by interfering with the VEGF- $\beta$-catenin-ERK signaling pathway [26]. Downregulation of miR-23b triggered glioma growth inhibition, induced apoptosis, and suppressed invasion by inhibition of $\beta$-catenin/Tcf4 and HIF-1 $\alpha /$ VEGF signaling pathways [27]. Moreover, miRNA-184 downregulated $\beta$-catenin and deleteriously changed endothelial cell adherens junction structure and functions in HUVECs and HCE [28] (Table 1).

\section{PECAM-1}

PECAM-1 is specifically expressed in blood and endothelial cells. PECAM-1 on circulating platelets and leukocytes functions as an inhibitory receptor that limits cellular activation responses. However, PECAM-1 is highly expressed at endothelial cell intercellular junctions, where it functions as a mechanical sensor, regulator of leukocyte trafficking, and in the maintenance of endothelial junction integrity [29]. Inhibition of miR-155 improved neurological impairment of rats with cerebral infarction accompanying enhancement of PECAM-1 expression and EC junction integrity improvement [30]. Interestingly, polyphenolics inhibited high glucosemediated inflammatory response and decreased PECAM-1 and ICAM-1 protein levels in human vascular endothelial cells (HUVECs). MicroRNA screens indicated that miR126 may be modulated by polyphenolics as the underlying mechanism to inhibit expression of PECAM-1 and ICAM1 [31]. A hyperoxia-induced bronchopulmonary dysplasia study resulted in lower levels of PECAM-1 mRNA and protein. MicroRNA screening indicated miRNAs may participate in the occurrence and development of bronchopulmonary dysplasia, including PECAM-1 expression. However, the underlying mechanism of miRNA-mediated PECAM-1 regulation and clinical consequence has not yet been determined [32] (Table 1).

\section{Focal Adhesion Junction Proteins}

5.1. Integrin $\beta 4$. In EC focal adhesion junctions, the major components include integrin $\beta 4$, paxillin, and focal adhesion kinase (FAK). Integrin $\beta 4$ associates with integrin $\alpha 6$ to form a complex which anchors ECs on the basal membrane and functions as a mechanical sensor. Unlike other integrin $\beta$ isoforms, integrin $\beta 4$ has a unique long cytosolic domain which functions as a dock for tyrosine kinases, such as Src and Fny. Once integrin $\beta 4$ receives stimulation from the basal membrane, its cytosolic domain recruits a tyrosine kinase and activates downstream signaling pathway. Singlenucleotide polymorphisms (SNPs) studies in breast cancer (BC) samples indicated that an A allele of the SNP rs 743554 in the integrin $\beta 4$ gene has strong association with oestrogen receptor-negative tumors and it is believed to affect binding efficiency with microRNAs. Compared with wild type genotype carriers, those with the A allele have a poor survival significantly associated with aggressive tumor characteristics: high grade, lymph node metastasis, and high stage [33]. MicroRNAs miR-184 and miR-205 competitively bind to the complementary sequences within the $3^{\prime}$ untranslated region ( $3^{\prime}$ UTR) of integrin $\beta 4$ mRNA. Mutated miR-184 failed to compete with miR-205 for the overlapping target sites on the $3^{\prime}$ UTRs of integrin $\beta 4$ and resulted in familial severe keratoconus [34]. MiR-205 also regulated tumor cell basal membrane deposits of lamin-332 and its receptor integrin $\beta 4$ 
[35]. Silico analysis showed that miR-9 also regulated integrin $\beta 4$ mRNA but had no experimental results validating it [36] (Table 1).

5.2. Paxillin. Knockdown of paxillin or ectopic expression of miR-137 inhibited tumor growth and metastasis of colorectal cancer (CRC) cells in vivo. A dual-luciferase reporter gene assay validated paxillin as a direct target of miR-137 [37]. A similar inhibitory effect of miR-137 was also observed in a non-small cell lung cancer model [38]. MiR-145 also inhibited paxillin expression by binding to the paxillin mRNA $3^{\prime} U T R$. Higher expression of paxillin and lower expression of miR145 were observed in colorectal cancer tissues than corresponding paracancerous tissue [39]. Among high-risk human papillomavirus (HPV) 16-infected oral cavity squamous cell carcinoma (OCSSC), HPV16/18 infection was negatively associated with miR-218 expression and positively associated with paxillin expression. This observation indicated there is an inverse relationship between miR-218 and paxillin in HPVinfected OCSCC [40]. More interestingly, microRNAs have been found to modulate paxillin expression by epigenetic mechanisms [41, 42]. Alternatively, paxillin also functions as a regulator of microRNA expression, such as miR-125b, to further regulate tumorigenesis [43] (Table 1).

5.3. Focal Adhesion Kinase (FAK). Focal adhesion kinase (FAK) is the key component of the focal adhesion complex, which links focal adhesions to the cytosolic signaling pathway. By modification of FAK, such as phosphorylation or dephosphorylation of tyrosine or serine, FAK transduces extracellular stimuli into downstream signaling, further regulating the cytoskeleton and cellular junction. miR-7 has been shown directly targeting FAK and is negatively correlated with lymph node metastasis and tumor node metastasis staging in colon cancer (CC) $[44,45]$. miR-543 level decreased in endometrial cancer cells and inversely correlated with mRNA levels of FAK and Twist homolog 1 (TWIST1) [46]. By binding with the $3^{\prime}$ UTR of mRNA, miR-138 and miR-135 inhibited FAK protein expression in different cancer cell lines, such as HeLa cell, SW480, and A375 cell. Moreover, regulation of FAK expression by miR-135 and miR-138 also affected cancer cell invasion, drug sensitivity, and tumor growth in both in vitro and in vivo models [47]. Finally, by targeting an upstream kinase of FAK, some miRNAs indirectly regulate FAK phosphorylation status and further affect cell functions, such as junction integrity, cell growth, and migration [48] (Table 1).

\section{Summary}

Junction proteins are groups of structural proteins involved in various critical cellular processes, including cellular connection and signal communication, cell growth, and migration. So far, endothelial junction integrity has shown to be involved in many diseases, such as edema, inflammatory cell infiltration, and tumor cell invasion. Accordingly, comprehensive understanding of cell junction protein regulation is crucial for both junction function study and clinical therapy. Among all of the regulatory mechanisms of junction protein expression, miRNAs have emerged as particularly promising targets for their structural and functional characteristics. In this review, we systemically reviewed the miRNAs and their targeting proteins of tight junctions, adherens junctions, focal adhesion junctions, and PECAM-1 junctions. Although some miRNAs -junction protein regulatory relationships were observed in models other than endothelial junctions, they still provide insights into understanding the importance of these miRNAs (Table 1). Moreover, the phenotypes of these regulatory miRNAs on targeting junction proteins are also presented and certainly help us better understand the importance of these miRNAs in clinical regards.

\section{Competing Interests}

The authors declare that they have no competing interests.

\section{Authors' Contributions}

Yugang Zhuang and $\mathrm{Hu}$ Peng equally contribute to this study.

\section{Acknowledgments}

This work was supported by the National Scientific Foundation of China (NSFC-81671938, Hu Peng; NSFC-81270133, Weiguo Chen).

\section{References}

[1] J. Amiel, L. de Pontual, and A. Henrion-Caude, "MiRNA, development and disease," Advances in Genetics, vol. 80, pp. 136, 2012.

[2] A. Henrion-Caude, M. Girard, and J. Amiel, "MicroRNAs in genetic disease: rethinking the dosage," Current Gene Therapy, vol. 12, no. 4, pp. 292-300, 2012.

[3] C. Cichon, H. Sabharwal, C. Rüter, and M. A. Schmidt, "MicroRNAs regulate tight junction proteins and modulate epithelial/endothelial barrier functions," Tissue Barriers, vol. 2, no. 4, Article ID e944446, 2014.

[4] W. Liu, H. Cai, M. Lin et al., "MicroRNA-107 prevents amyloidbeta induced blood-brain barrier disruption and endothelial cell dysfunction by targeting Endophilin-1," Experimental Cell Research, vol. 343, no. 2, pp. 248-257, 2016.

[5] Z. Fang, Q. He, Q. Li et al., "MicroRNA-150 regulates bloodbrain barrier permeability via Tie- 2 after permanent middle cerebral artery occlusion in rats," The FASEB Journal, vol. 30, no. 6, pp. 2097-2107, 2016.

[6] C. Rajput, M. Tauseef, M. Farazuddin et al., "MicroRNA-150 suppression of angiopoetin-2 generation and signaling is crucial for resolving vascular injury," Arteriosclerosis, Thrombosis, and Vascular Biology, vol. 36, no. 2, pp. 380-388, 2016.

[7] X. Ge, Z. Han, F. Chen et al., "MiR-21 alleviates secondary blood-brain barrier damage after traumatic brain injury in rats," Brain Research, vol. 1603, pp. 150-157, 2015.

[8] D. Hu, Y. Yu, C. Wang, D. Li, Y. Tai, and L. Fang, "microRNA98 mediated microvascular hyperpermeability during burn shock phase via inhibiting FIH-1," European Journal of Medical Research, vol. 20, no. 1, article 51, 2015. 
[9] Y. Wang and H. Yan, "MicroRNA-126 contributes to Niaspan treatment induced vascular restoration after diabetic retinopathy," Scientific Reports, vol. 6, Article ID 26909, 2016.

[10] J. Ma, Y. Yao, P. Wang et al., "MiR-181a regulates blood-tumor barrier permeability by targeting Krüppel-like factor 6," Journal of Cerebral Blood Flow \& Metabolism, vol. 34, no. 11, pp. 18261836, 2014.

[11] Y.-S. Miao, Y.-Y. Zhao, L.-N. Zhao et al., "MiR-18a increased the permeability of BTB via RUNX1 mediated down-regulation of ZO-1, occludin and claudin-5," Cellular Signalling, vol. 27, no. 1, pp. 156-167, 2015.

[12] W. Zhou, M. Y. Fong, Y. Min et al., "Cancer-Secreted miR-105 destroys vascular endothelial barriers to promote metastasis," Cancer Cell, vol. 25, no. 4, pp. 501-515, 2014.

[13] R. Mishra and S. K. Singh, "HIV-1 Tat C modulates expression of miRNA-101 to suppress VE-cadherin in human brain microvascular endothelial cells," Journal of Neuroscience, vol. 33, no. 14, pp. 5992-6000, 2013.

[14] E. Dejana, "Endothelial cell-cell junctions: happy together," Nature Reviews Molecular Cell Biology, vol. 5, no. 4, pp. 261-270, 2004.

[15] Y. Khew-Goodall and G. J. Goodall, "Myc-modulated miR-9 makes more metastases," Nature Cell Biology, vol. 12, no. 3, pp. 209-211, 2010.

[16] J. Qu, D. Lu, H. Guo, W. Miao, G. Wu, and M. Zhou, "MicroRNA-9 regulates osteoblast differentiation and angiogenesis via the AMPK signaling pathway," Molecular and Cellular Biochemistry, vol. 411, no. 1-2, pp. 23-33, 2016.

[17] N. M. Kane, L. Howard, B. Descamps et al., "Role of microRNAs 99b, 181a, and 181b in the differentiation of human embryonic stem cells to vascular endothelial cells," Stem Cells, vol. 30, no. 4, pp. 643-654, 2012.

[18] E. Di Bernardini, P. Campagnolo, A. Margariti et al., "Endothelial lineage differentiation from induced pluripotent stem cells is regulated by microRNA-21 and transforming growth factor $\beta 2$ (TGF- $\beta 2$ ) pathways," The Journal of Biological Chemistry, vol. 289, no. 6, pp. 3383-3393, 2014.

[19] F. Muramatsu, H. Kidoya, H. Naito, S. Sakimoto, and N. Takakura, "microRNA-125b inhibits tube formation of blood vessels through translational suppression of VE-cadherin," Oncogene, vol. 32, no. 4, pp. 414-421, 2013.

[20] M. K. Lalwani, M. Sharma, A. R. Singh et al., "Reverse genetics screen in zebrafish identifies a role of miR-142a-3p in vascular development and integrity," PLoS ONE, vol. 7, no. 12, Article ID e52588, 2012.

[21] J. A. Young, K. K. Ting, J. Li et al., "Regulation of vascular leak and recovery from ischemic injury by general and VE-cadherinrestricted miRNA antagonists of miR-27," Blood, vol. 122, no. 16, pp. 2911-2919, 2013.

[22] Y. Wang, B. Sun, H. Sun et al., "Regulation of proliferation, angiogenesis and apoptosis in hepatocellular carcinoma by miR-26b-5p," Tumor Biology, vol. 37, no. 8, pp. 10965-10979, 2016.

[23] K. Zhu, Q. Pan, L.-Q. Jia et al., "MiR-302c inhibits tumor growth of hepatocellular carcinoma by suppressing the endothelialmesenchymal transition of endothelial cells," Scientific Reports, vol. 4, article 5524, 2014.

[24] A. C. P. Correia, J.-R. A. J. Moonen, M. G. L. Brinker, and G. Krenning, "FGF2 inhibits endothelial-mesenchymal transition through microRNA-20a-mediated repression of canonical TGF- $\beta$ signaling," Journal of Cell Science, vol. 129, no. 3, pp. 569579, 2016.
[25] M. I. Almeida, R. M. Reis, and G. A. Calin, "MYC-microRNA9-metastasis connection in breast cancer," Cell Research, vol. 20, no. 6, pp. 603-604, 2010.

[26] H. Hirata, Y. Hinoda, K. Ueno, K. Nakajima, N. Ishii, and R. Dahiya, "MicroRNA-1826 directly targets beta-catenin (CTNNB1) and MEK1 (MAP2K1) in VHL-inactivated renal cancer," Carcinogenesis, vol. 33, no. 3, pp. 501-508, 2012.

[27] L. Chen, L. Han, K. Zhang et al., "VHL regulates the effects of miR-23b on glioma survival and invasion via suppression of HIF-1 $\alpha$ /VEGF and $\beta$-catenin/Tcf- 4 signaling," Neuro-Oncology, vol. 14, no. 8, pp. 1026-1036, 2012.

[28] R. Zong, T. Zhou, Z. Lin et al., "Down-regulation of MicroRNA184 is associated with corneal neovascularization," Investigative Opthalmology \& Visual Science, vol. 57, no. 3, pp. 1398-1407, 2016.

[29] J. R. Privratsky and P. J. Newman, "PECAM-1: regulator of endothelial junctional integrity," Cell and Tissue Research, vol. 355, no. 3, pp. 607-619, 2014.

[30] Y.-C. Meng, Z.-Y. Ding, H.-Q. Wang, L.-P. Ning, and C. Wang, "Effect of microRNA-155 on angiogenesis after cerebral infarction of rats through AT1R/VEGFR2 pathway," Asian Pacific Journal of Tropical Medicine, vol. 8, no. 10, pp. 829-835, 2015.

[31] G. D. Noratto, G. Angel-Morales, S. T. Talcott, and S. U. Mertens-Talcott, "Polyphenolics from Açaí (Euterpe oleracea Mart.) and red muscadine grape (Vitis rotundifolia) protect human umbilical vascular endothelial cells (HUVEC) from glucose- and lipopolysaccharide (LPS)-induced inflammation and target microRNA-126," Journal of Agricultural and Food Chemistry, vol. 59, no. 14, pp. 7999-8012, 2011.

[32] Y. Xing, J. Fu, H. Yang et al., "MicroRNA expression profiles and target prediction in neonatal Wistar rat lungs during the development of bronchopulmonary dysplasia," International Journal of Molecular Medicine, vol. 36, no. 5, pp. 1253-1263, 2015.

[33] A. Brendle, H. Lei, A. Brandt et al., "Polymorphisms in predicted microRNA-binding sites in integrin genes and breast cancer: ITGB4 as prognostic marker," Carcinogenesis, vol. 29, no. 7, pp. 1394-1399, 2008.

[34] A. E. Hughes, D. T. Bradley, M. Campbell et al., "Mutation altering the miR-184 seed region causes familial keratoconus with cataract," The American Journal of Human Genetics, vol. 89, no. 5, pp. 628-633, 2011.

[35] P. Gandellini, V. Profumo, A. Casamichele et al., " $m i R-205$ regulates basement membrane deposition in human prostate: implications for cancer development," Cell Death \& Differentiation, vol. 19, no. 11, pp. 1750-1760, 2012.

[36] W. Chen, M. C. Harbeck, W. Zhang, and J. R. Jacobson, "MicroRNA regulation of integrins," Translational Research, vol. 162, no. 3, pp. 133-143, 2013.

[37] D.-L. Chen, D.-S. Wang, W.-J. Wu et al., "Overexpression of paxillin induced by miR-137 suppression promotes tumor progression and metastasis in colorectal cancer," Carcinogenesis, vol. 34, no. 4, pp. 803-811, 2013.

[38] Y. Bi, Y. Han, H. Bi, F. Gao, and X. Wang, "miR-137 impairs the proliferative and migratory capacity of human non-small cell lung cancer cells by targeting paxillin," Human Cell, vol. 27, no. 3, pp. 95-102, 2014.

[39] J. Qin, F. Wang, H. Jiang, J. Xu, Y. Jiang, and Z. Wang, "MicroRNA-145 suppresses cell migration and invasion by targeting paxillin in human colorectal cancer cells," International Journal of Clinical and Experimental Pathology, vol. 8, no. 2, pp. 1328-1340, 2015. 
[40] D.-W. Wu, C.-Y. Chuang, W.-L. Lin, W.-W. Sung, Y.-W. Cheng, and H. Lee, "Paxillin promotes tumor progression and predicts survival and relapse in oral cavity squamous cell carcinoma by microRNA-218 targeting," Carcinogenesis, vol. 35, no. 8, pp. 1823-1829, 2014.

[41] D. Li, Z. Li, J. Xiong et al., "MicroRNA-212 functions as an epigenetic-silenced tumor suppressor involving in tumor metastasis and invasion of gastric cancer through downregulating PXN expression," American Journal of Cancer Research, vol. 5, pp. 2980-2997, 2015.

[42] J. Qin, J. Ke, J. Xu et al., "Downregulation of microRNA-132 by DNA hypermethylation is associated with cell invasion in colorectal cancer," OncoTargets and Therapy, vol. 8, pp. 36393648, 2015.

[43] A. Sen, H. Prizant, A. Light et al., "Androgens regulate ovarian follicular development by increasing follicle stimulating hormone receptor and microRNA-125b expression," Proceedings of the National Academy of Sciences of the United States of America, vol. 111, no. 8, pp. 3008-3013, 2014.

[44] X. Kong, G. Li, Y. Yuan et al., "MicroRNA-7 inhibits epithelialto-mesenchymal transition and metastasis of breast cancer cells via targeting FAK expression," PLoS ONE, vol. 7, no. 8, Article ID e41523, 2012.

[45] C.-Y. Zeng, Y.-S. Zhan, J. Huang, and Y.-X. Chen, "MicroRNA7 suppresses human colon cancer invasion and proliferation by targeting the expression of focal adhesion kinase," Molecular Medicine Reports, vol. 13, no. 2, pp. 1297-1303, 2016.

[46] L. Bing, C. Hong, S. Li-Xin, and G. Wei, "MicroRNA-543 suppresses endometrial cancer oncogenicity via targeting FAK and TWIST1 expression," Archives of Gynecology and Obstetrics, vol. 290, no. 3, pp. 533-541, 2014.

[47] V. M. Golubovskaya, B. Sumbler, B. Ho, M. Yemma, and W. G. Cance, "MiR-138 and MiR-135 directly target focal adhesion kinase, inhibit cell invasion, and increase sensitivity to chemotherapy in cancer cells," Anti-Cancer Agents in Medicinal Chemistry, vol. 14, no. 1, pp. 18-28, 2014.

[48] A. M. Mahmoud, W. Yang, and M. C. Bosland, "Soy isoflavones and prostate cancer: a review of molecular mechanisms," Journal of Steroid Biochemistry and Molecular Biology, vol. 140, pp. 116-132, 2014. 


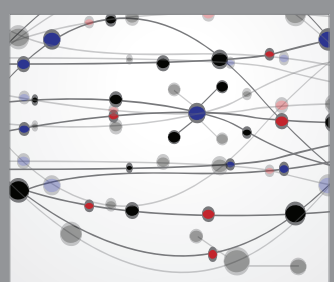

The Scientific World Journal
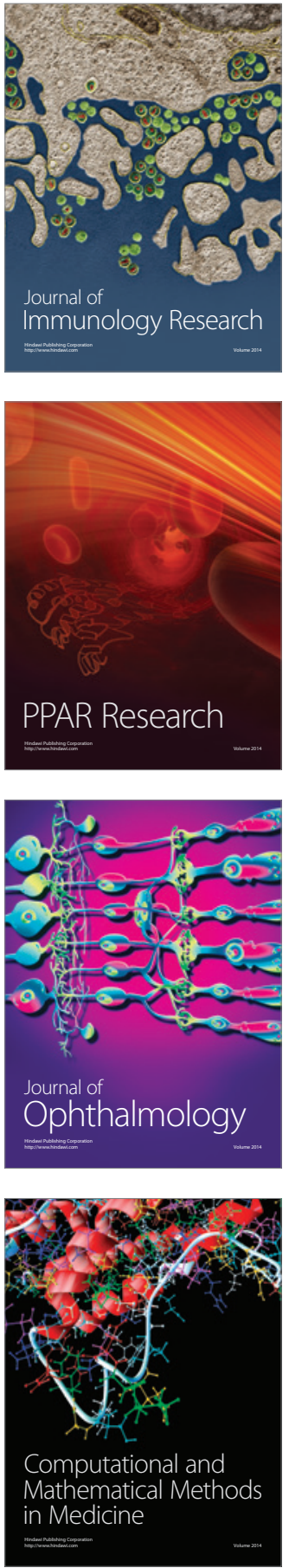

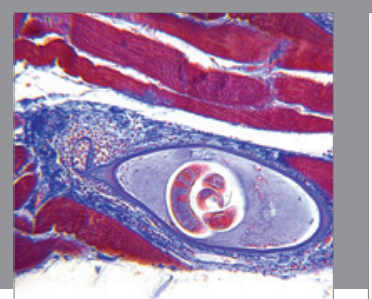

Gastroenterology Research and Practice

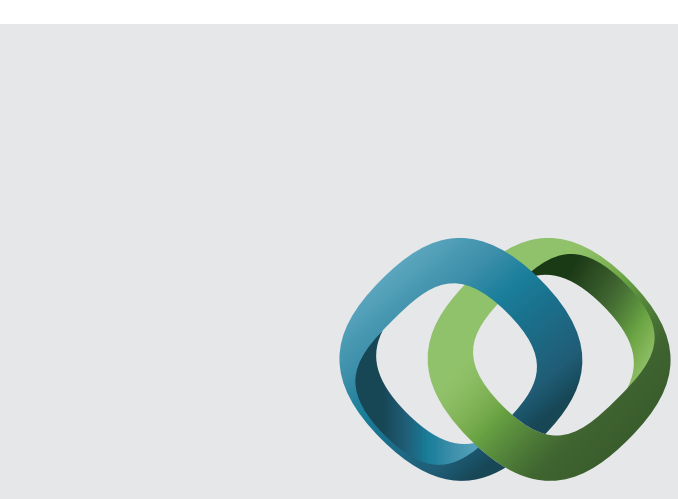

\section{Hindawi}

Submit your manuscripts at

http://www.hindawi.com
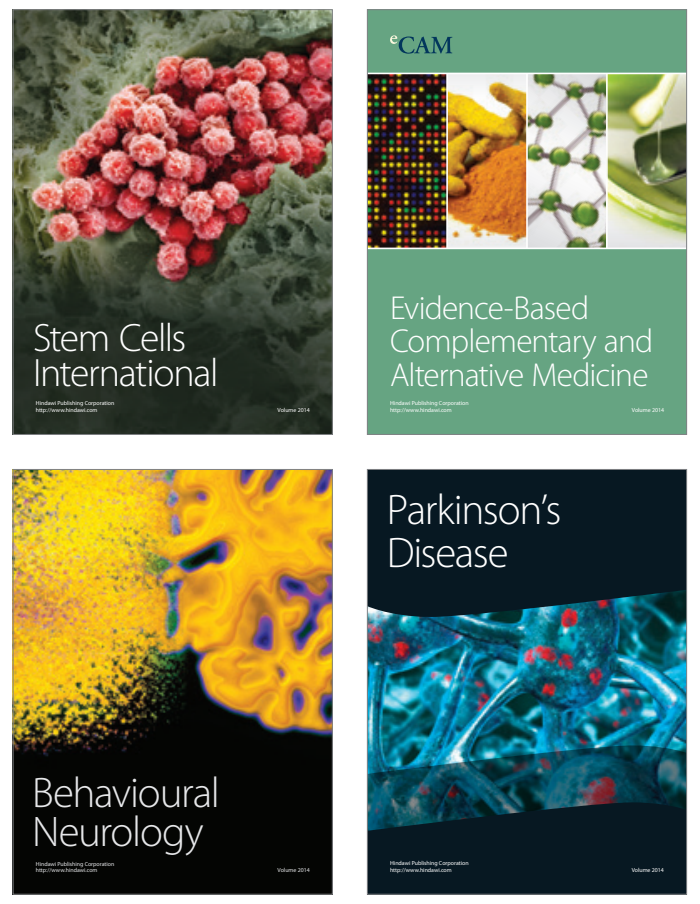
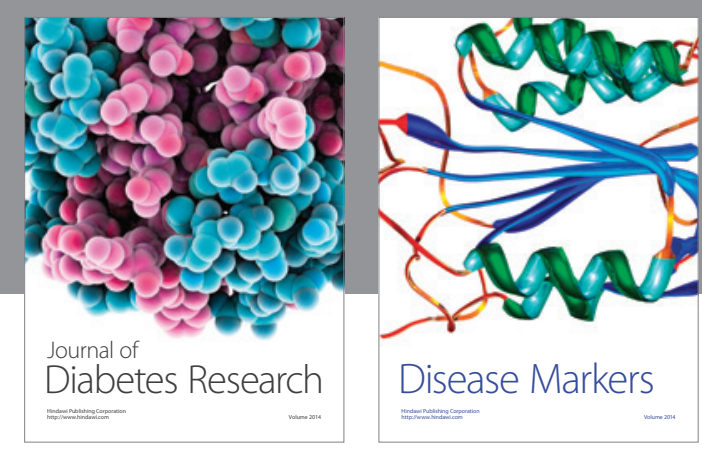

Disease Markers
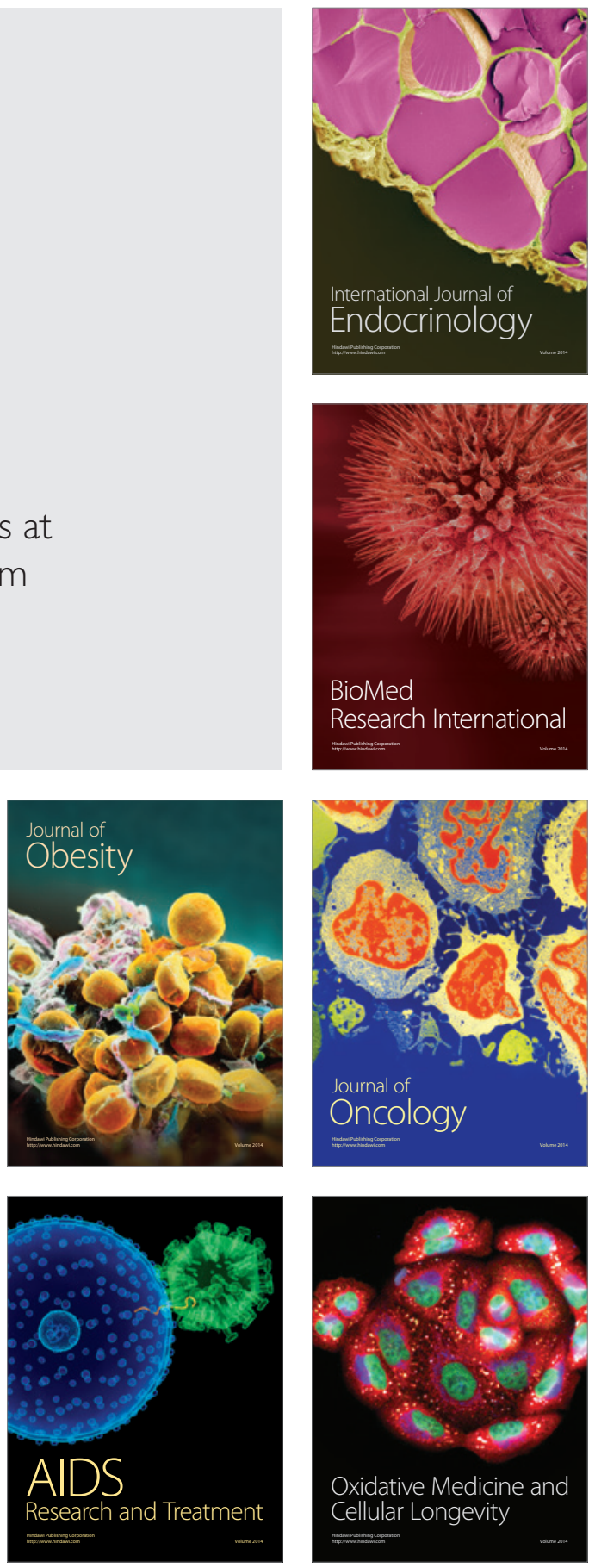